\title{
Tumour suppressor gene (CDKNA2) status on chromosome 9p in resected renal tissue improves prognosis of localised kidney cancer
}

\author{
Ismail El-Mokadem ${ }^{1}$, Thomas Kidd ${ }^{3}$, Norman Pratt ${ }^{2}$, Stewart Fleming ${ }^{3}$, Ghulam Nabi ${ }^{1}$ \\ ${ }^{1}$ Academic Section of Urology, Division of Cancer Research, University of Dundee, Ninewells Hospital, DD1 9SY, Dundee, Scotland \\ ${ }^{2}$ Department of Cytogenetic, University of Dundee, Ninewells Hospital, DD1 9SY, Dundee, Scotland \\ ${ }^{3}$ Department of Pathology, University of Dundee, Ninewells Hospital, DD1 9SY, Dundee, Scotland \\ Correspondence to: Ghulam Nabi, email: g.nabi@dundee.ac.uk
}

Keywords: kidney cancer, genetics, microsatellite analysis, chromosome $9 p$

Received: May 16, 2016

Accepted: September 15, 2016

Published: September 22, 2016

\section{ABSTRACT}

Background: Genetic alterations on chromosome 9p, including inactivation of the tumour suppressor gene, CDKN2A, result in cellular proliferation and growth of tumours. Our aim was to use microsatellite analysis and fluorescence in situ hybridization (FISH) to characterise the architecture of this region.

Results: Seventy-five out of $\mathbf{7 7}$ clear cell renal cell cancers (tumour/normal pairs) were interpretable for LOH analysis on chromosome 9p (two tumours were excluded, as all five primers were uninformative). Twenty out of $75(26.6 \%)$ tumours showed $\mathrm{LOH}$ in at least one of the five primers employed. Most allelic deletions were detected, telomeric to the CDKN2A region at D9S916, with 11 out of 52 informative tumours $(21 \%)$ displaying LOH. The LOH in the coding region of CDKN2A, at D9S974 and D9S942, was associated with a higher PT-stage $(p=0.004)$ and metastasis $(p=0.006$, both markers). The rate of chromosome 9p deletion in ccRCC was $44 \%$ ( $35 / 80$ cases) according to FISH. Somatic copy number loss of chromosome $9 \mathrm{p}$ was associated with a larger tumour size $(p=0.002)$, higher pathological tumour stage $(p=0.021)$, presence of tumour necrosis $(p=0.019)$ and microvascular invasion $(p=0.032)$. The cases with copy number loss, loss of heterozygosity and copy number neutral $(n=42)$ were at a higher risk of cancer-specific death when compared to tumours in category $D(n=32)$ (Log-rank: $p=0.001$ ). Seventeen patients with localised ccRCC developed recurrence, and fourteen of those showed either LOH or somatic copy number loss at CDKN2A (Log-rank: $p=0.005$ ). Multivariate analysis showed that LOH or copy number loss at CDKN2A retained its independent prognostic effect, improving the predictive accuracy of stage and SSIGN score by concordance Index C from 0.823 to $0.878(p=0.001)$.

Materials and Methods: Cytogenetics data, microsatellite analysis and FISH were acquired for a cohort of patients undergoing resection for clinically localised renal cancer between January 2001 and December 2005. Five microsatellite markers (D9S916, D9S1814, D9S974, D9S942 and D9S171) assessed loss of heterogeneity (LOH) using DNA samples and in the same cohort FISH analysis was accomplished on tissue microarray slides. The FISH data were scored by two observers blinded to the histological data of the patients. Cytogenetic aberrations were correlated with histological and clinical outcomes by univariate and multivariate analyses using different prognostic models. Disease specific and recurrence free survival based on cytogenetic changes were assessed by Kaplan Meier methods.

Conclusions: A comprehensive cytogenetic analysis using microsatellite analysis and FISH of the CDKN2A region on chromosome $9 \mathrm{p}$ improves the predictive accuracy of known prognostic factors in clinically localised renal cell carcinoma undergoing surgical resection. 


\section{INTRODUCTION}

Loss of heterozygosity (LOH) and copy number deletion are two different cytogenetic aberrations which can result in down-regulation or inactivation of a gene. These two mechanisms could be involved in tumour progression if they result in inactivation of tumour suppressor genes, such as CDKN2A/B located on chromosome 9p.

CDKNA region contains genetic information for the synthesis of many tumour suppressor proteins such as $\mathrm{p} 16^{\mathrm{INK} 4 \mathrm{a}}, \mathrm{p} 14^{\mathrm{ARF}}$, and $\mathrm{p} 15^{\mathrm{INK} 4 \mathrm{~b}}$. These proteins regulate progression of cellular proliferation through cell cycle, for example p16INK4a plays a key role in transition of cell from G1-S phase in the cell cycle through p16-Rb pathway. In addition, this plays an active role in cellular senescence, p16 through interconnections influences tumor suppressor P53 pathways $[1,2]$ Any alteration in this region (mutation or hypermethylation) can potentially lead to reduced or no synthesis of these proteins and hence unregulated growth of cells [3].

Several cytogenetic methods, such as fluorescence in situ hybridization (FISH), comparative genomic hybridization $(\mathrm{CGH})$ and microsatellite analysis, have been reported; each has its advantages and disadvantages. A recent review of published literature on prognostic value of 9p deletion in RCC has shown that most studies were observational and used a wide variety of molecular and conventional cytogenetic techniques. The studies were mostly old series with no common protocols, which led to a discrepancy in the outcomes and the quality of the studies reviewed [4].

Three studies have shown that somatic copy number loss involving $9 \mathrm{p}$ can be associated independently with worse outcomes in ccRCC. All three studies relied on I-FISH to assess loss of chromosome 9p; however, the criteria and cut-off values used to define deletion differed among the studies, as did the probes employed [5-7]. Our group used I-FISH to carry out a validation study and confirmed the above findings in patients with surgically resected RCC that had the longest follow-up [8]. The probe employed was a dual-colour probe with a locusspecific identifier on the CDKN2A gene.

By contrast, studies using microsatellite analysis have demonstrated that $\mathrm{LOH}$ on chromosome $9 \mathrm{p}$ is associated with adverse histological features and a risk of progression of renal cancer [9-11]. Microsatellite analysis, using paired control normal renal tissue and tumour DNA, is a sensitive technique for detecting $\mathrm{LOH}$ in tumours. It is a fast and inexpensive analytical method and can be performed on degraded DNA extracted from formalin-fixed, paraffin-embedded tissue. Kinoshita et al. were the first to propose that $\mathrm{LOH}$ in the region of $9 \mathrm{p} 21$ was associated with progression of RCC and metastasis [9], but the study lacked long-term follow-up. Only a few studies correlated the allelic deletion of $9 p$ and survival with varied follow-up duration which ranged between 31 and 48 months [11-13]. Some studies relied on only one or two microsatellites telomeric to $9 \mathrm{p} 21$ for assessment of allelic deletion of $9 p$ and could therefore have missed more centromeric $\mathrm{LOH}$ involving regions harbouring several tumour suppressor genes involved in cell cycle regulation.

In comparison to I-FISH, microsatellite karyotyping detects $\mathrm{LOH}$ in the absence of copy number variation (deletion). This phenomenon is also known as copy number neutral LOH (CNNLOH) and was previously called uniparental disomy (UPD). Studies on oesophageal cancer have shown that copy number neutral $\mathrm{LOH}$ is a common phenomenon $[14,15]$ The literature contains few reports on CNN-LOH in clear cell RCC especially in the region of chromosome $9 \mathrm{p} 21$, which harbours $\mathrm{CDKN} 2 \mathrm{~A} / \mathrm{B}$, one of the main tumour suppressor genes. $\mathrm{CNN}-\mathrm{LOH}$ could therefore be an underestimated event which could result in gene inactivation due to duplication of the mutated or non-functioning allele.

The objective of the present study were:

(i) to assess the concordance between microsatellites and I-FISH, including the diagnostic utility of the individual methods.

(ii) to determine whether the overall prognostic impact of a $9 p$ deletion could be improved using a strategy incorporating FISH and $\mathrm{LOH}$ analysis, particularly in conjunction with long-term follow-up.

\section{RESULTS}

\section{Cohort characteristics}

The study population included 89 (82.4\%) patients with clear cell histology, $13(12 \%)$ with papillary renal cell carcinoma (pRCC), 5 (4.6\%) with chromophobe appearance and one (1\%) with collecting duct carcinoma. The clear cell subtype was the focus of this study; therefore, somatic copy number loss and LOH in the $9 p$ region were analysed in relation to adverse histopathological factors and survival only in this subtype. However, we included all the subtypes in the concordance analysis of $\mathrm{LOH}$ between microsatellites and I-FISH. The mean age of patients with ccRCC was 62.8 (range $38-84$ ) with a mean follow-up of 80 months (range 6-165 months).

\section{Microsatellite analysis of $\mathrm{LOH}$ at the 9p21 region and correlation with pathological parameters in ccRCC}

In total, 96 out of 108 cases represented on the tissue microarrays (TMAs) were tested for LOH with 5 markers. Four markers (D9S916, D9S974, D9S942 and D9S1814) were within the 9p21 region and 1 marker (D9S171) was within 9p13 region. Seventy-seven (80\%) tumours 
were clear cell subtype, 13 (13.5\%) were papillary RCC, $5(5.2 \%)$ were chromophobe RCC, and only one was collecting duct RCC.

Except for two uninformative cases, 75 out of 77 ccRCC tumour/normal pairs were interpretable for $\mathrm{LOH}$ analysis on chromosome 9p (Figure 1). Average tumour size for these patient was $6.3 \mathrm{~cm}$. The mean age in this cohort was 62.5 years old. The median follow-up was 91.8 months (mean 85 months). Again, details of allelic deletions have been reported previously [16].

In addition to previous publication, $\mathrm{LOH}$ at D9S916 was statistically associated with a higher pT stage $(p=0.005)$, sarcomatoid changes $(p=0.026)$, and renal sinus invasion $(p=0.014)$, with only an observed trend for metastasis $(p=0.10)$.

The LOH within the coding region of CDKN2A, at D9S974 and D9S942, was also associated with higher pT stage ( $p=0.004$ and $p=0.003$, respectively and metastasis ( $p=0.006$, both markers). The LOH within D9S974 and D9S942 was also associated with renal sinus invasion ( $p=0.015$ and $p=0.006$, respectively.

Almost half the cases were uninformative at D9S1814, but the LOH at this locus was associated only with higher $\mathrm{pT}$ stage $(p=0.028)$. A trend was also observed with renal sinus invasion $(p=0.059)$.

On the other hand, LOH was the only marker within 9p13 (D9S171) and showed a correlation with renal sinus invasion only $(p=0.027)$. A trend was also noted with pathological tumour stage $(p=0.083)$.

\section{Fluorescence in-situ hybridisation and correlation with pathological parameters}

Of the 108 tumours, 98 had valid 9p scoring on the TMA. Eighty of these 98 cases were ccRCC subtype and were included in the survival analysis in this study. Twelve were papillary RCC, five were chromophobe RCC and only one was a collecting duct carcinoma.

A complete description of the results of this experiment was previously reported $[8,7]$. Based on I-FISH interpretation using pre-set criteria, the rate of chromosome 9 p deletion in ccRCC was 44\% (35/80 cases). Somatic copy number loss of chromosome $9 \mathrm{p}$ was associated with a larger tumour size $(p=0.002)$, a higher pathological tumour stage $(p=0.021)$, the presence of tumour necrosis $(p=0.019)$ and microvascular invasion $(p=0.032)$.

\section{Concordance between LOH on microsatellite analysis and fluorescence in-situ hybridization in all RCC specimens}

Ninety-six cases (paired tumour and normal tissue) were assessed for microsatellite analysis and included in the concordance analysis for 9p loss using FISH.

Of the 68 cases with informative markers within the coding region of CDKN2A (D9S974 and D9S942), LOH was observed in 17 tumours $(25.7 \%)$, with 7 tumours showing $\mathrm{LOH}$ at both microsatellites and 10 tumours showing LOH for one of the two markers.

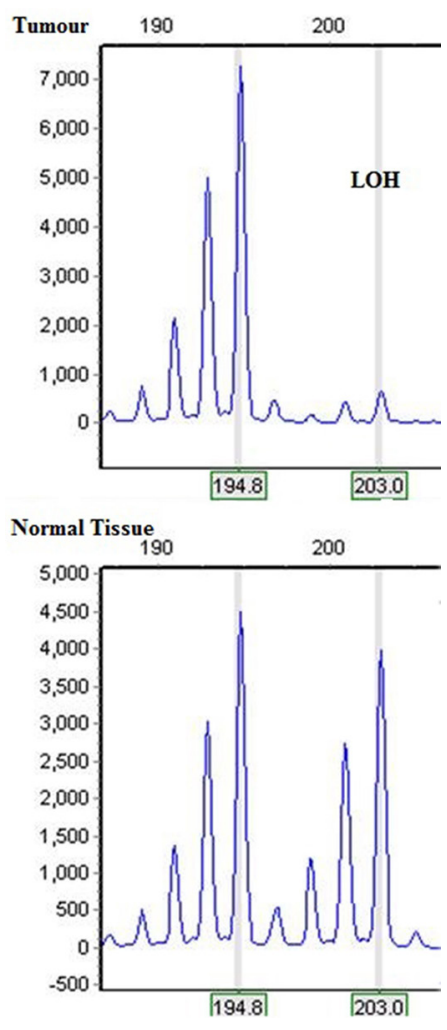

Figure 1: Microsatellite analysis is showing allelic deletion in tumour 81 involving more than one marker. 
Table 1: Concordance between FISH and microsatellite analyses in the region of CDKN2A

\begin{tabular}{lcccc}
\hline \multicolumn{2}{c}{$\begin{array}{c}\text { Concordance } \\
\text { Analysis }\end{array}$} & \multicolumn{3}{c}{ I-FISH } \\
\cline { 3 - 5 } & No & No Deletion & Monosomy/LOH & Total \\
\hline \multirow{2}{*}{$\begin{array}{l}\text { LOH analysis with } \\
\text { microsatellites }\end{array}$} & Yes & 30 & 14 & 44 \\
& Total & 9 & 6 & 15 \\
& & 39 & 20 & 59 \\
\hline
\end{tabular}

Sixty-three of the 68 informative cases had valid I-FISH scores available for comparison. Four tumours were excluded from concordance analysis as they were marked as a homozygous loss by I-FISH. In total, 59 tumours were available for concordance analysis between microsatellites and I-FISH. Both techniques agreed on 30 tumours showing no $\mathrm{LOH}$ or copy number loss at CDKN2A. LOH within CDKN2A region was detected and agreed upon by both the techniques in 6 tumours. Overall, the concordance rate between microsatellites and I-FISH was $61 \%(36 / 59)$.

On the other hand, microsatellite analysis showed LOH at CDKN2A in 9 tumours that did not show copy number loss on FISH. This finding could be explained by the common phenomenon of copy number neutral $\mathrm{LOH}(\mathrm{CNNLOH})$. The rate of CNNLOH at the CDKN2A coding region was $60 \%(9 / 15)$.

A further analysis of 14 tumours showing $9 p$ loss using I-FISH, with an average pooled percentage of abnormal nuclei of $49 \%$ (range: $39-60 \%$ ), did not show allelic loss on microsatellite analysis. As a result, the sensitivity of microsatellite analysis to detect copy number loss LOH was poor $(30 \% ; 6 / 20)$ when compared to FISH.

\section{Combined copy number loss and loss of heterozygosity on chromosome 9p and its impact on prognosis in ccRCC}

The data of copy number loss using FISH and from microsatellite analysis on the $\mathrm{LOH}$ in the $\mathrm{CDKN} 2 \mathrm{~A}$ region were combined to assess the impact of aberrations on the outcomes of ccRCC. Seventy-four tumours with complete information were divided into four categories:
A. Tumours with somatic copy number loss $(n=29)$.
B. Tumours with somatic copy number loss and LOH $(n=6)$.
C. Tumours with copy number neutral LOH $(n=7)$.
D. Tumours with normal copy number and heterozygous $(n=32)$.

The cases in the first three groups combined $(n=42)$ were at a higher risk of cancer-specific death when compared to tumours in category $\mathrm{D}(n=32)$ (Figure 2: Log-rank: $p=0.001)$. Seventeen patients with localised ccRCC developed recurrence; fourteen of these showed either LOH or somatic copy number loss at CDKN2A (Figure 3: Log-rank: $p=0.005$ ).
Univariate Cox-proportional hazard analysis revealed that cases with $\mathrm{LOH}$ or copy number loss at CDKN2A showed a higher hazard ratio when compared to each aberration independently for disease-specific survival (DSS) and recurrence-free survival (RFS) (Table 1). All cases were categorised based on $\mathrm{LOH}$ status and copy number loss at $\mathrm{CDKN} 2 \mathrm{~A}$ in relation to validated prognostic nomograms for metastasis or RFS and DSS in ccRCC (Table 2).

Multivariate analysis for DSS revealed that $\mathrm{LOH}$ or copy number loss at CDKN2A retained its independent prognostic effect with pathological $\mathrm{T}$ stage and state of metastasis in model 1 (Table 3). It also remained as an independent prognostic factor with the Mayo Clinical Stage, Size, Grade and Necrosis (SSIGN) score in model 2 , improving its predictive accuracy expressed by concordance Index C from 0.823 to $0.878(p=0.001)$.

On the other hand, multivariate analysis for RFS showed that combination of copy number loss or $\mathrm{LOH}$ at $\mathrm{CDKN} 2 \mathrm{~A}$ is an independent prognostic factor for recurrence in ccRCC in addition to the pT stage in model 3 (Table 4). The integration of copy number loss or $\mathrm{LOH}$ at CDKN2A with the Leibovich score enhanced its predictive accuracy expressed by concordance Index $\mathrm{C}$ from 0.734 to $0.801(p=0.009)$.

\section{DISCUSSION}

This study has confirmed previous findings where the LOH on chromosome 9p in clear cell RCC is associated with adverse histopathological features and worse prognosis, but it also showed enhanced prognostic significance when LOH is combined with FISH analysis.

The detection rate of allelic deletion-as previously reported- on chromosome 9p in ccRCC by [16] studies using microsatellites $[10,12,13]$ and more contemporary studies relying on SNP arrays [21, 22].

The LOH within the coding region of CDKN2A was associated with higher tumour grade and a higher risk of metastasis. The frequency of $\mathrm{LOH}$ for all 4 markers on 9p21 (D9S916, D9S974, D9S942 and D9S1814) was significantly associated with locally advanced tumours, highlighting our previous observation that $\mathrm{LOH}$ could be a biomarker for aggressive renal cell cancer. The LOH on $9 \mathrm{p} 21$, especially the markers within the region of CDKN2A in addition to D9S916, were all independent prognostic factors in multivariate analysis models when 
Table 2: Univariate Cox proportional hazard model

\begin{tabular}{|c|c|c|c|c|c|}
\hline \multicolumn{6}{|c|}{ Univariate analysis for disease-specific survival (DSS) } \\
\hline \multirow{2}{*}{ Variable } & \multirow{2}{*}{ Categories } & \multirow{2}{*}{$p$-value } & \multirow{2}{*}{ Hazard Ratio } & \multicolumn{2}{|c|}{$95 \%$ CI } \\
\hline & & & & Lower & Upper \\
\hline LOH at CDKN2A & Yes vs. No & 0.005 & 5.374 & 1.644 & 17.564 \\
\hline $\begin{array}{l}\text { Somatic deletion at } \\
\text { CDKN2A }\end{array}$ & Yes vs. No & 0.002 & 5.703 & 1.904 & 17.077 \\
\hline $\begin{array}{l}\text { Combined deletion or } \\
\text { LOH at CDKN2A }\end{array}$ & $\begin{array}{c}\text { Deletion or LOH vs. } \\
\text { normal }\end{array}$ & 0.004 & 8.464 & 1.958 & 36.578 \\
\hline \multicolumn{6}{|c|}{ Univariate analysis for recurrence-free survival (RFS) } \\
\hline \multirow{2}{*}{ Variable } & \multirow{2}{*}{ Categories } & \multirow{2}{*}{$p$-value } & \multirow{2}{*}{ Hazard Ratio } & \multicolumn{2}{|c|}{$95 \% \mathrm{CI}$} \\
\hline & & & & Lower & Upper \\
\hline LOH at CDKN2A & Yes vs. No & 0.023 & 4.040 & 1.213 & 13.460 \\
\hline $\begin{array}{l}\text { Somatic deletion at } \\
\text { CDKN2A }\end{array}$ & Yes vs. No & 0.003 & 5.365 & 1.763 & 16.327 \\
\hline $\begin{array}{l}\text { Combined deletion or } \\
\text { LOH at CDKN2A }\end{array}$ & $\begin{array}{c}\text { Deletion or LOH vs. } \\
\text { normal }\end{array}$ & 0.007 & 5.620 & 1.670 & 32.674 \\
\hline
\end{tabular}

integrated separately for DSS. In the group of clinically localised ccRCC, the $\mathrm{LOH}$ in the CDKN2A region was associated with a higher risk of recurrence or development of metastatic disease, as determined by univariate analysis ( $p=0.023$; HR 4.04). This LOH remained an independent prognostic factor in the multivariate analysis model including the Leibovich score $(p=0.014$; HR $=4.7)$.
These findings confirm again that $\mathrm{LOH}$ involving chromosome 9p21, and particularly LOH at the coding region of CDKN2A, portends a worse prognosis in ccRCC in long term follow up and they validated the findings from I-FISH-based analysis of 9p deletion.

Previous studies have suggested that the $\mathrm{LOH}$ on chromosome $9 \mathrm{p}$ is associated with adverse histological

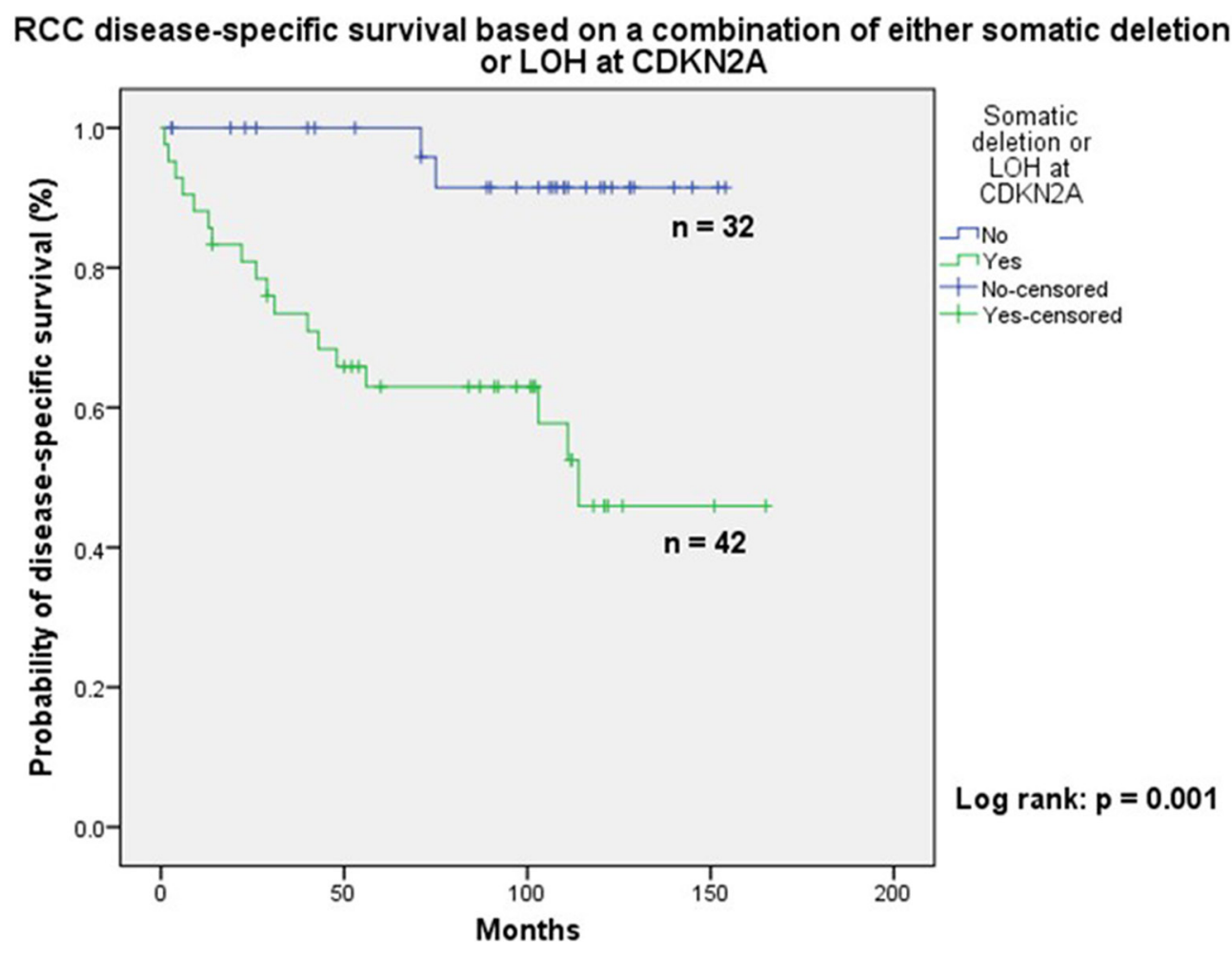

Figure 2: Kaplan Meir analysis clearly showing poor disease specific survival in patients with cytogenetic abnormalities on chromosome 9p. 
Table 3: Association between copy number loss and LOH on CDKN2A with SSIGN and Leibovich scores

\begin{tabular}{llcccccc}
\hline & & $\begin{array}{c}\text { Copy number loss at } \\
\text { CDKN2A }\end{array}$ & \multicolumn{2}{c}{$\begin{array}{c}\text { LOH at } \\
\text { CDKN2A }\end{array}$} & $\begin{array}{c}\text { LOH or copy number loss at } \\
\text { CDKN2A }\end{array}$ \\
\hline \multirow{3}{*}{ SSIGN score } & \multicolumn{2}{c}{ Deletion } & No deletion & LOH & Normal & Yes & No \\
\hline & Low (0-3) & 10 & 25 & 5 & 28 & 13 & 19 \\
& Intermediate (4-7) & 15 & 13 & 5 & 18 & 17 & 8 \\
& High (8-13) & 10 & 7 & 3 & 8 & 12 & 5 \\
Leibovich & Total & $\mathbf{3 5}$ & $\mathbf{4 5}$ & $\mathbf{1 3}$ & $\mathbf{5 4}$ & $\mathbf{4 2}$ & $\mathbf{3 2}$ \\
score & Low (0-2) & 5 & 20 & 2 & 21 & 7 & 16 \\
& Intermediate (3-5) & 12 & 13 & 6 & 17 & 15 & 8 \\
& High (6-11) & 12 & 8 & 2 & 13 & 12 & 6 \\
& Total & $\mathbf{2 9}$ & $\mathbf{4 1}$ & $\mathbf{1 0}$ & $\mathbf{5 1}$ & $\mathbf{3 4}$ & $\mathbf{3 0}$ \\
\hline
\end{tabular}

features and risk of progression of renal cancer $[11,9,10]$. Kinoshita et al. were the first to propose that $\mathrm{LOH}$ in the region of 9p21 was associated with progression of $\mathrm{RCC}$ and metastasis [9], but the study lacked long-term followup. Our literature search revealed several studies that reported on allelic deletion on chromosome $9 \mathrm{p}$ in renal cancer, but only a few studies established correlations between allelic deletion of $9 p$ and survival with sufficient follow-up periods $[11,13]$. Some of the studies relied on only one or 2 microsatellites telomeric to 9 p21 to assess allelic deletion of $9 \mathrm{p}$, which could have missed more centromeric LOH involving regions harbouring several tumour suppressor genes involved in cell cycle regulation. Allelic deletion of 9p was assessed by 5 markers covering the 9 p21 region, as the region of interest that harbours CDKN2A/B genes, and to validate I-FISH results, which relied on a dual-colour probe with a locus-specific identifier on CDKN2A.

The follow-up period in the previous studies ranged between 31 and 48 months (median), compared to 91 months median follow-up in this study. The extended follow-up period for this cohort allowed assessment of the impact of LOH on 9p21, and especially the coding region of CDKN2A, on survival. The prognostic impact of $\mathrm{LOH}$

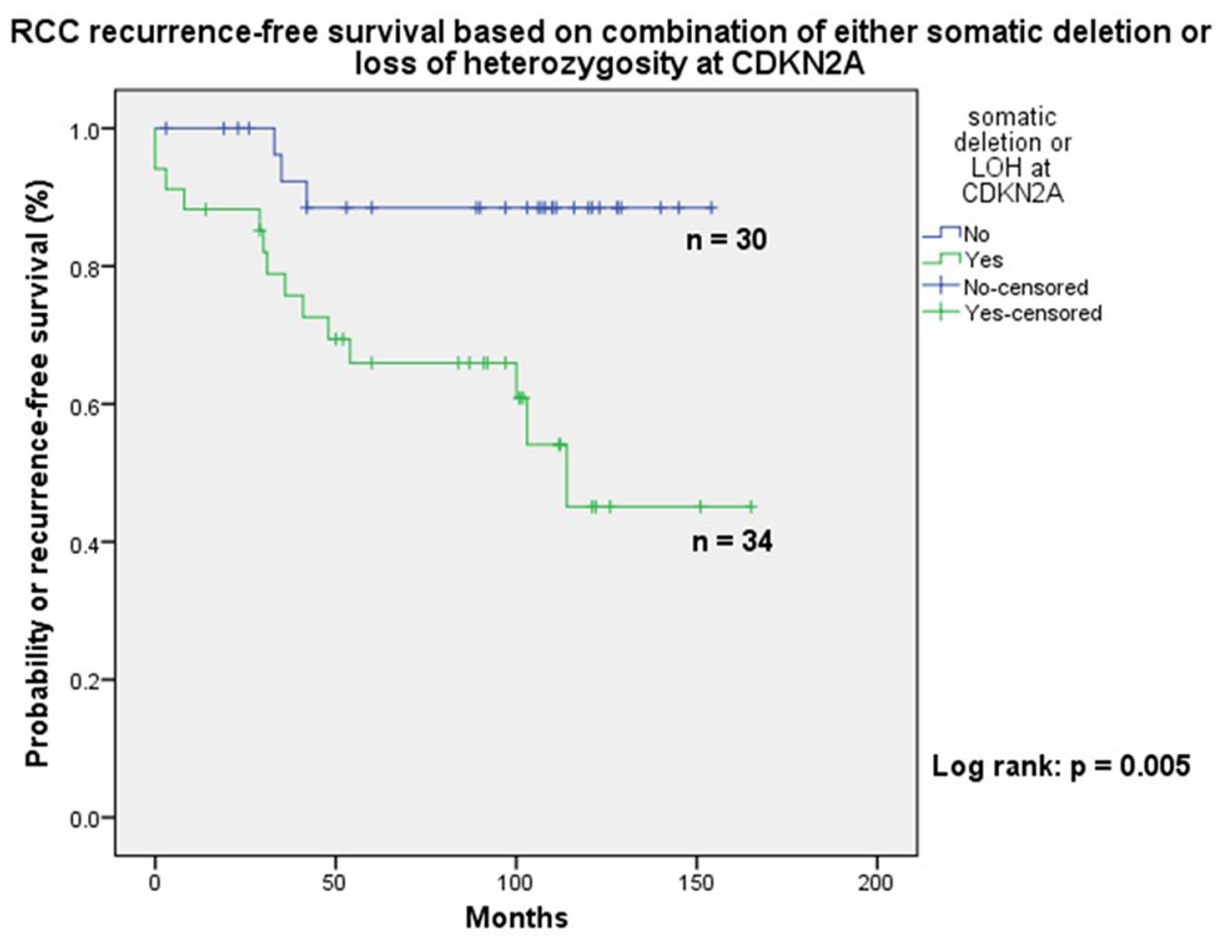

Figure 3: Showing better recurrence free survival in patients with no loss of heterozygosity or deletion of chromosome 9p. 
Table 4: Multivariate Cox proportional hazard model analysis for RFS and DSS

\begin{tabular}{|c|c|c|c|c|c|}
\hline \multicolumn{6}{|c|}{ Model 1 for RCC disease-specific survival (DSS) } \\
\hline \multirow{2}{*}{ Variable } & \multirow{2}{*}{ Categories } & \multirow{2}{*}{$p$-value } & \multirow{2}{*}{$\begin{array}{c}\text { Hazard } \\
\text { Ratio }\end{array}$} & \multicolumn{2}{|c|}{$95 \% \mathrm{CI}$} \\
\hline & & & & Lower & Upper \\
\hline $\begin{array}{l}\text { Combined deletion or } \mathrm{LOH} \text { at } \\
\text { CDKN2A }\end{array}$ & $\begin{array}{l}\text { Deletion or LOH vs. } \\
\text { normal }\end{array}$ & 0.045 & 4.568 & 1.031 & 20.238 \\
\hline T-stage & pT3/4 vs. pT1/2 & 0.006 & 6.724 & 1.710 & 26.435 \\
\hline Metastasis & $\mathrm{N}+\mathrm{M}+$ vs. N0M0 & 0.003 & 4.532 & 1.678 & 12.241 \\
\hline \multicolumn{6}{|c|}{ Model 2 for RCC disease-specific survival (DSS) } \\
\hline \multirow{2}{*}{ Variable } & \multirow{2}{*}{ Categories } & \multirow{2}{*}{$p$-value } & \multirow{2}{*}{$\begin{array}{c}\text { Hazard } \\
\text { Ratio }\end{array}$} & \multicolumn{2}{|c|}{$95 \% \mathrm{CI}$} \\
\hline & & & & Lower & Upper \\
\hline $\begin{array}{l}\text { Combined deletion or } \mathrm{LOH} \text { at } \\
\text { CDKN2A }\end{array}$ & $\begin{array}{c}\text { Deletion or LOH vs. } \\
\text { normal }\end{array}$ & 0.010 & 6.808 & 1.567 & 29.567 \\
\hline SSIGN score & 3 sub-categories & $<0.001$ & 4.361 & 2.163 & 8.974 \\
\hline \multicolumn{6}{|c|}{ Model 3 for recurrence-free survival (RFS) } \\
\hline \multirow{2}{*}{ Variable } & \multirow{2}{*}{ Categories } & \multirow{2}{*}{$p$-value } & \multirow{2}{*}{$\begin{array}{c}\text { Hazard } \\
\text { Ratio }\end{array}$} & \multicolumn{2}{|c|}{$95 \% \mathrm{CI}$} \\
\hline & & & & Lower & Upper \\
\hline T-stage & pT3/4 vs. pT1/2 & 0.007 & 4.188 & 1.491 & 11.761 \\
\hline $\begin{array}{l}\text { Combined deletion or } \mathrm{LOH} \text { at } \\
\text { CDKN2A }\end{array}$ & $\begin{array}{c}\text { Deletion or LOH vs. } \\
\text { normal }\end{array}$ & 0.023 & 5.701 & 1.265 & 25.701 \\
\hline \multicolumn{6}{|c|}{ Model 4 for recurrence-free survival (RFS) } \\
\hline \multirow{2}{*}{ Variable } & \multirow{2}{*}{ Categories } & \multirow{2}{*}{$p$-value } & \multirow{2}{*}{$\begin{array}{c}\text { Hazard } \\
\text { Ratio }\end{array}$} & \multicolumn{2}{|c|}{$95 \% \mathrm{CI}$} \\
\hline & & & & Lower & Upper \\
\hline $\begin{array}{l}\text { Combined deletion or } \mathrm{LOH} \text { at } \\
\text { CDKN2A }\end{array}$ & $\begin{array}{c}\text { Deletion or LOH vs. } \\
\text { normal }\end{array}$ & 0.019 & 5.968 & 1.347 & 26.435 \\
\hline Leibovich score & 3 sub-categories & 0.006 & 3.061 & 1.387 & 6.758 \\
\hline
\end{tabular}

on $9 p$ was determined by integrating it within multivariate models including the most significant variables and in externally validated prognostic models for cancer-specific survival and metastasis-free survival.

Microsatellite analysis has the advantage of detecting loss of heterozygosity in the absence of copy number variation (deletion), which is also known as copy number neutral LOH (CNNLOH). Studies on several cancers have shown that CNNLOH is a common phenomenon, but its correlation with gene expression remains poorly understood. It could still be considered to represent a mechanism of gene inactivation, especially if the wild type allele is lost and the non-functioning one is duplicated to maintain a copy neutral status $[14,15]$.

The incidence of CNNLOH has been studied in detail in oesophageal cancer, and it appeared to play a role in carcinogenesis and was associated with a change in gene expression levels, either by up-regulation or down- regulation. However, the authors concluded that the mechanism remains poorly understood $[14,15]$. The authors suggested that $\mathrm{CNNLOH}$ was more common than
LOH with copy number alteration in oesophageal cancer and represented $70 \%$ of all allelic deletions detected by single nucleotide polymorphism (SNP) arrays. Saeki et al. reported copy neutral LOH of the p53 locus in p53 mutant oesophageal cancer and suggested that this could be a major mechanism for inactivation of the intact allele in oesophageal squamous cell carcinogenesis associated with a mutation at $\mathrm{p} 53$. The authors suggested five scenarios that could result in LOH on $\mathrm{p} 53$; three of them are copy neutral [15].

The present study revealed CNNLOH in 9 tumours. As expected, these were not detected by I-FISH alone and hence alternate/complementary techniques are required to map these areas. On the contrary, copy number loss on chromosome $9 \mathrm{p}$ was detected by I-FISH alone but not by microsatellite analysis. This discordance can be explained by a number of factors; for example, contamination of tumour samples during macro-dissection (viable tumour areas were marked by a specialist pathologist on tumour blocks in this study); intratumour heterogeneity causing contamination of DNA predominantly from a sub clone with no copy number loss; or a high percentage of homozygous loss or DNA 
sample contamination with normal renal tissue. The lack of sensitivity of microsatellite analysis in reference to FISH to detect copy number loss LOH comes down predominantly to the superiority of FISH to cut through tumour complexity at a cellular level, allowing the observer to score regions of tumour in more than one core that has a high concentration of cells with copy number loss. This advantage is clearly lost in microsatellite analysis as it relies on DNA extracted from a macro-dissected tumour region, resulting in dilution of abnormality by a mixture of normal cells and tumour cells without copy number loss of chromosome 9p. Previous work has shown that if the proportion of tumour cells in a sample diminishes to $50 \%$ or less, the ability to detect the $\mathrm{LOH}$ is reduced [23]. Moreover, the discordance could have been the result of sampling bias; I-FISH results were based on the scoring of 6 different cores in contrast to three 8-10 $\mu \mathrm{m}$ curls used for DNA extraction for microsatellite analysis. The increase in the number of sampled tumour regions clearly improves the detection and identification of more subclones [24]. This could have been potentially prevented by laser microdissection of tumour tissue before DNA extraction to avoid contamination with normal renal tissue or necrotic tumour tissue, which is a potential limitation in our study.

The addition of copy number status to allelic deletion measured by $\mathrm{LOH}$ analysis at the CDKN2A coding region on chromosome $9 p$ significantly enhances the predictive accuracy of the SSIGN score and Leibovich score for cancer-specific death and development of metastasis in patients undergoing resection for clinically localised renal cell carcinoma. The assessment of copy number loss with I-FISH and LOH using microsatellites is complementary for the detection of copy number aberrations and provides better prognostication data. However, discordance still exists in a number of cases for reasons explained in this study.

In conclusion, combined cytogenetic techniques improve the prognostic significance of known factors in clinically localised renal cancer following surgery. Copy number neutral LOH, seen in $10 \%$ of the cohort, carries a worse prognosis and perhaps could be a potential candidate for closer follow-up or adjuvant therapy.

\section{MATERIALS AND METHODS}

\section{Study cohort}

The database of Tayside Urological Cancer Network (TUCAN) databases were searched for consecutive patients who underwent radical nephrectomy for Renal Cell Carcinoma (RCC) between Jan. 2001 and Dec. 2005. This allowed each participants of the study to have atleast 10 years of follow-up. This is same cohort described previously in detail for Fluorescent in situ hybridization and microsatellite alteration studies $[8,16]$. Ethical approval through local research committee (Ref. $12 / \mathrm{ES} / 0083$ ) was obtained prior to initiation of the study.
Patients were followed up using a standard protocol based on tumour stage and grade. All tumour characteristics, clinical and follow-up data were collated for each case. Tumours were examined and re-classified according to the 2009 TNM staging [17-19]. All cases were assigned a Mayo-Clinic SSIGN score [17] and only cases with localized ccRCC were assigned a Leibovich score [20].

Follow-up was calculated from the time of surgery to the last date of assessment or date of death. The cause of death was determined based on death certificates and correspondence between clinicians and patients' general practitioners. Death from renal cancer was defined as disease-related mortality. Recurrence was diagnosed if RCC metastasis or renal bed recurrence was detected on cross-sectional imaging and confirmed in a multidisciplinary team meeting record.

\section{Tissue microarray (TMA)}

This was described in previous publication from the same institution [7, 19]. In summary, TMAs were constructed using Beecher ${ }^{\circledR}$ arraying instrument (Beecher Instruments Inc., Sun Prairie, WI, USA) with the help of TMA Designer ${ }^{\circledR} 2$ software. Tissue cores with a diameter of $0.6 \mathrm{~mm}$ were punched from the marked tumour regions on paraffin blocks after being marked by the pathologist. Cores were then deposited into a master paraffin block and placed $1.2 \mathrm{~mm}$ apart from the neighbouring core on the $\mathrm{x}$ and $\mathrm{y}$-axes. Sections from the resulting master paraffin block measuring $4 \mu \mathrm{m}$ in thickness were then transferred onto glass slides to form a TMA. Each case was represented at least with six tumour cores. A TMA from normal renal tissue from the same cases was constructed to be used as a control to determine the cut-off threshold for deletion.

\section{Fluorescence in-situ hybridization (FISH)}

Interphase I-FISH analysis was performed using the Vysis Locus-Specific Identifier (LSI) CDKN2A spectrum red (R)/(CEP 9) spectrum green $(\mathrm{G})$ probes (Abbott Molecular, USA). The process of applying the probe to TMA slides is described in detail in our previous publication. Interpretation of I-FISH, using pre-set criteria, was carried out by 2 independent observers blinded to clinical outcomes to determine $9 p$ status in each tumour based on 6 representative cores on the TMA and relying on control normal tissue to set threshold for deletion. Any disagreement was settled by reviewing slides and consensus. An algorithm describing the interpretation of I-FISH for $9 \mathrm{p}$ deletion was published previously [8].

\section{DNA extraction from formalin-fixed paraffin-embedded tissue samples}

Three curls were cut from the same FFPE tumour blocks that were represented by six cores on the tumour 
TMAs. This was done to minimise the risk of sampling bias and reduce the effect of intratumour heterogeneity on the results.

DNA extraction was undertaken from FFPE tumour curls and corresponding normal tissue curls from the same patient using the automated EZ1 BioRobot method. This extraction protocol relies on the use of magnetic particles that bind to the DNA and are subsequently removed from the surrounding tissue by a separate magnetic source. As with the manual methods, $180 \mu \mathrm{l}$ ATL tissue lysis buffer was added to each sample $(2 \times 10 \mu \mathrm{M}$ FFPE sections $)$ together with $20 \mu$ l Proteinase K solution. The samples were placed in an Eppendorf Thermomixer and incubated at $56^{\circ} \mathrm{C}$ with vigorous shaking for one hour. At the end of this period, the thermomixer temperature was increased to $90^{\circ} \mathrm{C}$ and incubated another hour, again with vigorous shaking. The samples were then loaded into a cartridge and placed in the EZ1 Robot. All the reagents required for the extraction of DNA from a sample are present in a single cartridge. An elution volume of $50 \mu \mathrm{l}$ ATE buffer was used before DNA sample was stored at $-20^{\circ} \mathrm{C}$ until use. All DNA samples concentration and quality were assessed by NanoDrop 1000 spectrometer (Thermo Fisher Scientific, Wilmington, Del).

\section{Microsatellites markers}

Five fluorescent end-labelled microsatellite markers that map to the 9p13-21 region were used after being checked for possible single nucleotide polymorphisms (SNP). Four markers map to 9p21; D9S916, D9S1814, D9S974 and D9S942. The latter two are within the coding sequence of CDKN2A. D9S916 is telomeric to CDKN2A and CDKN2B, whereas D9S1814 is centromeric to CDKN2B. D9S171 is at 9p13 (proximal toCDKN2A/B). The details of these microsatellite markers have been described in our recent study [16].

PCR amplifications were performed in a final volume of 24ul and consisted of 10X PCR reaction buffer, $2 \mathrm{mM}$ dNTPs, forward and reverse primers, HotStarTaq DNA Polymerase ${ }^{\circledR}\left(\right.$ Qiagen $\left.^{\circledR}\right)$ and distilled H2O. All PCRs reagents were mixed in one tube, $24 \mu \mathrm{l}$ of the reaction mix was aliquoted into each tube pre-labelled with the sample number. The sample DNA was added (usually $1 \mu \mathrm{l}$; 50-200 ng) to each corresponding labelled tube and tubes placed in the thermal cycler.

The PCR programme employed consisted of 1 cycle at $95^{\circ} \mathrm{C}$ for 15 minutes for activation of HotStarTaq. This was followed by 35 cycles of denaturation step $\left(94^{\circ} \mathrm{C}\right.$ for 40 seconds), then annealing step $\left(55^{\circ} \mathrm{C}\right.$ for 40 seconds $)$, followed by elongation or extension step $\left(72^{\circ} \mathrm{C}\right.$ for 40 second). Finally the programme terminates with 1 cycle of cooling $\left(8^{\circ} \mathrm{C}\right.$ for 7 minutes, then $4^{\circ} \mathrm{C}$ for 1 minute)

Following amplification, $0.5 \mu \mathrm{l}$ of the product was denatured in deionized formamide and the fluorescent markers analysed by capillary electrophoresis. Determination of loss of heterozygosity (LOH) status was carried out by staff in the NHS Genetic Laboratory, using GeneMarker ${ }^{\text {B }}$ Software V2.4.0 (SoftGenetic ${ }^{\circledR}$, State College, PA 16803, USA). For informative cases, the allelic loss was scored if the signal intensity of one allele was reduced by more than $40 \%$ in the tumour DNA compared to the corresponding allele in normal DNA.

\section{Statistical analysis}

The clinicopathological data were compared based on $9 p$ status. Proportions between categorical variables were compared using Fisher's exact and Pearson chisquare tests, as appropriate. The survival time was summarised using mean and ranges. Other continuous variables were summarised as means and standard deviation (SD) and compared using Student- $t$ tests or Mann-Whitney $U$ test as appropriate.

The Kaplan-Meier method was used to estimate RFS and DSS based on 9p status and other variables. The log-rank test was used to compare the survival differences between the groups.

A univariate Cox proportional hazard model was used to assess the correlation between prognostic variables and recurrence, and RCC-specific mortality. Multivariate analysis was performed for DSS and RFS after excluding the insignificant variables in univariate analysis. Backwards selection manner with the likelihood ratio criterion (for entry and removal: $p \leq 0.05$ and $p>0.1$ respectively) and rank of elimination was used to identify the most significant variables to be entered in the final models for RFS and DSS. The predictive accuracy of prognostic models was assessed by employing Concordance index (C-index). Statistical analysis was performed using IBM $^{\circledR}$ SPSS $^{\circledR}$ - version 21, with all tests being two-tailed and $p<0.05$ considered statistically significant.

\section{CONFLICTS OF INTEREST}

None.

\section{REFERENCES}

1. Rayess H, Wang MB, Srivatsan ES. Cellular senescence and tumor suppressor gene p16. International journal of cancer. 2012; 130:1715-1725.

2. Harris SL, Levine AJ. The p53 pathway. positive and negative feedback loops. Oncogene. 2005; 24:2899-2908.

3. Nichols AC, Yoo J, Palma DA, Fung K, Franklin JH, Koropatnick J, Mymryk JS, Batada NN, Barrett JW. Frequent mutations in TP53 and CDKN2A found by nextgeneration sequencing of head and neck cancer cell lines. Archives of otolaryngology — head \& neck surgery. 2012; 138:732-739.

4. El-Mokadem I, Fitzpatrick J, Rai B, Cunningham J, Pratt N, Fleming S, Nabi G. Significance of chromosome 9p status in renal cell carcinoma: a systematic review and quality of 
the reported studies. BioMed research international. 2014; 2014:521380.

5. Brunelli M, Eccher A, Gobbo S, Ficarra V, Novara G, Cossu-Rocca P, Bonetti F, Menestrina F, Cheng L, Eble JN, et al. Loss of chromosome $9 \mathrm{p}$ is an independent prognostic factor in patients with clear cell renal cell carcinoma. Modern pathology. 2008; 21:1-6.

6. La Rochelle J, Klatte T, Dastane A, Rao N, Seligson D, Said J, Shuch B, Zomorodian N, Kabbinavar F, Belldegrun A, et al. Chromosome 9p deletions identify an aggressive phenotype of clear cell renal cell carcinoma. Cancer. 2010; 116:4696-4702.

7. de Oliveira D, Dall'Oglio MF, Reis ST, Zerati M, Souza IC, Leite KR, Srougi M. Chromosome 9p deletions are an independent predictor of tumor progression following nephrectomy in patients with localized clear cell renal cell carcinoma. Urologic oncology. 2014; 32:601-606.

8. El-Mokadem I, Fitzpatrick J, Bondad J, Rauchhaus P, Cunningham J, Pratt N, Fleming S, Nabi G; Chromosome $9 p$ deletion in clear cell renal cell carcinoma predicts recurrence and survival following surgery. British journal of cancer. 2014; 111:1381-1390.

9. Kinoshita H, Yamada H, Ogawa O, Kakehi Y, Osaka M, Nakamura E, Mishina M, Habuchi T, Takahashi R, Sugiyama T, et al. Contribution of chromosome 9p21-22 deletion to the progression of human renal cell carcinoma. Japanese journal of cancer research. 1995; 86:795-799.

10. Schullerus D, Herbers J, Chudek J, Kanamaru H, Kovacs G. Loss of heterozygosity at chromosomes $8 p, 9 p$, and $14 q$ is associated with stage and grade of non-papillary renal cell carcinomas. The Journal of pathology. 1997; 183:151-155.

11. Li X, Tan X, Yu Y, Chen H, Chang W, Hou J, Xu D, Ma L, Cao G. D9S168 microsatellite alteration predicts a poor prognosis in patients with clear cell renal cell carcinoma and correlates with the down-regulation of protein tyrosine phosphatase receptor delta. Cancer. 2011; 117:4201-4211.

12. Schraml P, Struckmann K, Bednar R, Fu W, Gasser T, Wilber K, Kononen J, Sauter G, Mihatsch MJ, Moch H. CDKNA2A mutation analysis, protein expression, and deletion mapping of chromosome $9 p$ in conventional clear-cell renal carcinomas: evidence for a second tumor suppressor gene proximal to CDKN2A. The American journal of pathology. 2001; 158:593-601.

13. Presti JC Jr, Wilhelm M, Reuter V, Russo P, Motzer R, Waldman F. Allelic loss on chromosomes 8 and 9 correlates with clinical outcome in locally advanced clear cell carcinoma of the kidney. The Journal of urology. 2002; 167:1464-1468.

14. Hu N, Clifford RJ, Yang HH, Wang C, Goldstein AM, Ding T, Taylor PR, Lee MP. Genome wide analysis of DNA copy number neutral loss of heterozygosity $(\mathrm{CNNLOH})$ and its relation to gene expression in esophageal squamous cell carcinoma. BMC genomics. 2010; 11:576.
15. Saeki H, Kitao H, Yoshinaga K, Nakanoko T, Kubo N, Kakeji Y, Morita M, Maehara Y. Copy-neutral loss of heterozygosity at the p53 locus in carcinogenesis of esophageal squamous cell carcinomas associated with p53 mutations. Clinical cancer research. 2011; 17:1731-1740.

16. El-Mokadem I, Lim A, Kidd T, Garret K, Pratt N, Batty D, Fleming S, Nabi G. Microsatellite alteration and immunohistochemical expression profile of chromosome 9 p21 in patients with sporadic renal cell carcinoma following surgical resection. BMC cancer. 2016; 16:546.

17. Ficarra V, Novara G, Galfano A, Brunelli M, Cavalleri S, Martignoni G, Artibani W. The Stage, Size, Grade and Necrosis' score is more accurate than the University of California Los Angeles Integrated Staging System for predicting cancer-specific survival in patients with clear cell renal cell carcinoma. BJU international. 2009; 103:165-170.

18. Sobin L GM, Wittekind C. TNM classification of malignant tumors. UICC International Union Against Cancer. In. Edited by UICC, 7 edn. USA: Wiley-Blackwel; 2009.

19. Ficarra V, Martignoni G, Lohse C, Novara G, Pea M, Cavalleri S, Artibani W. External validation of the Mayo Clinic Stage, Size, Grade and Necrosis (SSIGN) score to predict cancer specific survival using a European series of conventional renal cell carcinoma. The Journal of urology. 2006; 175:1235-1239.

20. Leibovich BC, Blute ML, Cheville JC, Lohse CM, Frank I, Kwon ED, Weaver AL, Parker AS, Zincke H. Prediction of progression after radical nephrectomy for patients with clear cell renal cell carcinoma: a stratification tool for prospective clinical trials. Cancer. 2003; 97:1663-1671.

21. Sato Y, Yoshizato T, Shiraishi Y, Maekawa S, Okuno Y, Kamura T, Shimamura T, Sato-Otsubo A, Nagae G, Suzuki H, et al. Integrated molecular analysis of clear-cell renal cell carcinoma. Nature genetics. 2013; 45:860-867.

22. Toma MI, Grosser M, Herr A, Aust DE, Meye A, Hoefling C, Fuessel S, Wuttig D, Wirth MP, Baretton GB. Loss of heterozygosity and copy number abnormality in clear cell renal cell carcinoma discovered by high-density affymetrix $10 \mathrm{~K}$ single nucleotide polymorphism mapping array. Neoplasia. 2008; 10:634-642.

23. Yamamoto G, Nannya Y, Kato M, Sanada M, Levine RL, Kawamata N, Hangaishi A, Kurokawa M, Chiba S, Gilliland DG, et al. Highly sensitive method for genomewide detection of allelic composition in nonpaired, primary tumor specimens by use of affymetrix singlenucleotide-polymorphism genotyping microarrays. American journal of human genetics. 2007; 81:114-126.

24. Gerlinger M, Horswell S, Larkin J, Rowan AJ, Salm MP, Varela I, Fisher R, McGranahan N, Matthews N, Santos CR, et al. Genomic architecture and evolution of clear cell renal cell carcinomas defined by multiregion sequencing. Nature genetics. 2014; 46:225-233. 